\title{
Asymptotic Analysis of the MMSE Multiuser Detector for Nonorthogonal Multipulse Modulation
}

\author{
Michael L. McCloud and Louis L. Scharf, Fellow, IEEE
}

\begin{abstract}
We develop the minimum mean-squared-error (MMSE) multiuser detector for nonorthogonal multipulse modulation over the noncoherent additive white Gaussian noise channel. We analyze the asymptotic performance of the detector and show that, unlike the case of linear modulation, the MMSE detector does not generally approach the generalized maximum-likelihood (GML) detection rule as the noise power vanishes. It does, however, approach a detector which nulls out the multiaccess interference. This detector is termed the multipulse decorrelating detector due to its similarity to the linear decorrelating detector. The probability of error for this detector is derived and used to find the asymptotic multiuser efficiencies of both the multipulse decorrelating detector and the MMSE detector. It is shown that for noncoherent binary signaling, in which the multipulse modulation is two-dimensional, the multipulse decorrelating detector is superior to the GML detector asymptotically. This result does not generalize to larger dimensionality signal sets.
\end{abstract}

Index Terms-Code-division multiple access, minimum mean squared error detection, multipulse modulation, multiuser detection, noncoherent detection.

\section{INTRODUCTION}

$\mathbf{O}$ RTHOGONAL signaling is often employed to communicate over noncoherent channels. However, when multiple users share such a channel, the assignment of mutually orthogonal signal sets to each user requires a large bandwidth. Moreover, if each user employs a signal set which is orthogonal but correlated with the other users, then a low complexity receiver may produce an effective signal constellation which is no longer orthogonal for each user. For these reasons, we consider the more general case of nonorthogonal multipulse modulation (NMM) in which each user is assigned a possibly correlated signal set; from which one signal is transmitted at each signaling period. There is also the possibility of bandwidth savings through the use of NMM. Orthogonal signaling schemes require a bandwidth which grows linearly with the number of signals employed, while NMM can generally be made much more spectrally efficient.

Zero-forcing (or decorrelative) detection of such signals has been studied recently in [1]-[5]. These detectors act to first remove the multiple-access interference (MAI) through a perpendicular projection of the received data out of the span of the

Paper approved by U. Mitra, the Editor for Spread Spectrum/Equalization of the IEEE Communications Society. Manuscript received September 9, 1999 revised March 30, 2000 and May 4, 2000. This work was supported by the National Science Foundation under Contract ECS 9979400 and by the Office of Naval Research under Contract N00014-00-0033. This paper was presented in part at the IEEE International Symposium on Information Theory, Sorrento, Italy, June 2000.

The authors are with the Department of Electrical and Computer Engineering, University of Colorado at Boulder, Boulder, CO 80309-0425 USA (e-mail: mccloud@ucsu.colorado.edu).

Publisher Item Identifier S 0090-6778(01)00253-7. interfering user's signals. This operation is followed by either the asymptotically optimal detector of [5] if the user's energies are available or by the generalized maximum-likelihood (GML) detector [1]-[5] in the absence of this information. In [6], the authors extended the subspace tracking techniques of [7] to develop a blind GML detector which employs subspace tracking to estimate the interfering users' subspace.

In this letter we consider the use of the minimum mean-squared-error (MMSE) rule for NMM communication. This detector was previously derived in [6] and it was noted that for the example therein, the GML detector appeared to outperform the MMSE detector asymptotically. In this letter we derive the asymptotic performance of the MMSE detector and compare it to that of the GML detector derived in [3]-[5]. We first show that unlike the case of linear modulation considered in [8], the MMSE detector does not approach the GML detector as the noise power vanishes, although it does approach a detector which completely nulls the MAI. This detector is termed the multipulse decorrelating (MD) detector, based on its similarity to the linear decorrelating detector of [9]. The two-signal version of this detector was independently proposed in [10], in a somewhat different context. It is shown that the MD and the MMSE behave differently from the GML detector for large values of the signal-to-noise ratio (SNR). While there does not seem to be any reason to prefer the MD or MMSE detector over the GML detector in general, we prove that the MD (and hence the MMSE) is asymptotically superior to the GML detector for binary signaling from a two-dimensional multipulse signal set. This result does not extend to higher dimensional signaling. The performance comparison between the MD and the MMSE detectors at low SNRs is still an open question. This question is similar in spirit to that addressed in [11] for coherent, linear, modulation.

\section{Discrete TIME MODEL}

The discrete time model for the output of the noncoherent channel with NMM is [6]

$$
\mathbf{y}=\mathcal{H D} \boldsymbol{b}+\mathbf{n} .
$$

The matrix $\mathcal{H}=[\mathbf{H}(1), \mathbf{H}(2), \ldots, \mathbf{H}(K)]$ contains the signal vectors for each user with $\mathbf{H}(k)=\left[\mathbf{h}_{1}(k), \mathbf{h}_{2}(k), \ldots, \mathbf{h}_{M}(k)\right]$ and $\mathbf{h}_{m}(k)$ is the $m$ th signal corresponding to user $k$. The vector $\boldsymbol{b}=\left[\mathbf{b}^{T}(1), \mathbf{b}^{T}(2), \ldots, \mathbf{b}^{T}(K)\right]^{T}$ is an $M K \times 1$ vector with each $\mathrm{b}(k)$ a column of the $M \times M$ identity matrix which selects the signal transmitted by user $k$. That is, $\mathbf{H}(k) \mathbf{b}_{m}(k)=\mathbf{h}_{m}(k)$. The $M K \times M K$ matrix

$$
\begin{gathered}
\mathcal{D}=\operatorname{diag}\left\{\sqrt{E_{1}} e^{j \theta_{1}(1)}, \sqrt{E_{1}} e^{j \theta_{2}(1)}, \ldots, \sqrt{E_{1}} e^{j \theta_{M}(1)}, \ldots,\right. \\
\left.\sqrt{E_{K}} e^{j \theta_{1}(K)}, \ldots, \sqrt{E_{K}} e^{j \theta_{M}(K)}\right\}
\end{gathered}
$$


contains the user energies and phase terms. The individual gain parameters, $\sqrt{E_{k}} e^{j \theta_{m}(k)}$ are modeled as having an amplitude, $\sqrt{E_{k}}$, which is independent of the transmitted symbol, but a phase, $\theta_{m}(k)$ which may be hypothesis dependent. The additive noise, $\mathbf{n}$, is modeled as zero-mean complex Gaussian with correlation matrix $E\left[\mathbf{n n}^{*}\right]=\sigma^{2} \mathbf{I}$.

Assuming that the phase terms are independent zero-mean random variables, the measurement $y$ has first and second order statistics

$$
\mathbf{m}=E[\mathbf{y}]=\mathbf{0} \quad \text { and } \quad \mathbf{R}=E\left[\mathbf{y} \mathbf{y}^{*}\right]=\frac{1}{M} \mathcal{H} \mathbf{F} \mathcal{H}^{*}+\sigma^{2} \mathbf{I}
$$

where $\mathbf{F}=\operatorname{diag}\left\{E_{1} \mathbf{I}, \ldots, E_{K} \mathbf{I}\right\}$.

We may expand this model when the $k$ th user is of interest and has transmitted signal $\mathbf{h}_{m}(k)$

$$
\mathbf{y}=\sqrt{E_{k}} e^{j \theta_{m}(k)} \mathbf{h}_{m}(k)+\mathbf{S}(k) \beta+\mathbf{n}
$$

where the matrix $\mathbf{S}(k) \in \mathbb{C}^{N \times M(K-1)}$ is formed from the matrices $\mathbf{H}(l)$ for $l \neq k$ and $\beta \in \mathbb{C}^{M(K-1) \times 1}$ is formed by stacking the vectors $\sqrt{E_{l}} e^{j \theta_{m_{l}}} \mathbf{b}(k)$. We assume that the users communicate independently.

\section{MMSE DETECTOR}

The MMSE estimator of the vector $\mathcal{D} \boldsymbol{b}$ is given by

$$
\widehat{\mathcal{D} b}=\mathbf{F} \mathcal{H}^{*} \mathbf{R}^{-1} \mathbf{y}
$$

where $\mathbf{F}, \mathcal{H}$, and $\mathbf{R}$ are defined in Section II. If we consider the $k$ th block of $\widehat{\mathcal{D} \boldsymbol{b}}, \mathbf{D}(\widehat{k) \mathbf{b}}(k)$, we obtain a simple decision rule for the noncoherent channel

$$
\begin{aligned}
\hat{m}_{\mathrm{MMSE}}(k) & =\arg \max _{m} \mid\left\{\left.\mathbf{D}(\widehat{k) \mathbf{b}}(k)\}_{m}\right|^{2}\right. \\
& =\arg \max _{m}\left\{\left|\mathbf{h}_{m}^{*}(k) \mathbf{R}^{-1} \mathbf{y}\right|^{2}\right\} .
\end{aligned}
$$

This is motivated by the fact that the true vector $\mathbf{D}(k) \mathrm{b}(k)$ has the form

$$
\mathrm{D}(k) \mathbf{b}(k)=\left[\begin{array}{lllllll}
0 & \cdots & 0 & \mu_{m}(k) & 0 & \cdots & 0
\end{array}\right]^{T}
$$

i.e., it is nonzero only in the $m$ th position when symbol $m$ is transmitted by user $k$.

Geometrically, we see that the noncoherent detector seeks the whitened signal vector, $\mathbf{R}^{-1 / 2} \mathbf{h}_{m}(k)$, which is closest to the whitened measurement, $\mathbf{R}^{-1 / 2} \mathbf{y}$, in terms of the magnitude squared inner product. This MMSE detector chooses the maximum of a bank of $M$ whitened, noncoherent, matched filters.

\section{MD DETECTOR}

For NMM, the noncoherent GML detector is given by [1]-[5]

$$
\hat{m}_{\mathrm{GML}}(k)=\arg \max _{m} \frac{\left|\mathbf{h}_{m}^{*}(k) \mathbf{P}_{\mathbf{S}(k)}^{\perp} \mathbf{y}\right|^{2}}{\mathbf{h}_{m}^{*}(k) \mathbf{P}_{\mathbf{S}(k)}^{\perp} \mathbf{h}_{m}(k)}
$$

where $\mathbf{P}_{\mathbf{S}(k)}^{\perp}$ is the orthogonal projection matrix with null space $\langle\mathbf{S}(k)\rangle,{ }^{1}$ the so-called MAI space. For the case of linear, co-

\footnotetext{
${ }^{1}\langle\mathbf{A}\rangle$ denotes the subspace spanned by the columns of the matrix $\mathbf{A}$.
}

herent, modulation, the MMSE detector is known to approach the GML (decorrelating) detector as the noise power vanishes [8]. However, the following theorem shows that the MMSE detector is not generally asymptotically equivalent to the GML detector for NMM, although they are both zero-forcing, resulting in complete MAI removal.

Theorem IV.1: The MMSE detector is asymptotically given by

$$
\begin{aligned}
& \hat{m}_{\mathrm{MD}}(k) \\
& \quad=\arg \max _{m}\left|\left\{\left(\mathbf{H}^{*}(k) \mathbf{P}_{\mathbf{S}(k)}^{\perp} \mathbf{H}(k)\right)^{+} \mathbf{H}(k)^{*} \mathbf{P}_{\mathbf{S}(k)}^{\perp} \mathbf{y}\right\}\right|_{m}^{2}
\end{aligned}
$$

where the superscript + denotes the pseudoinverse, so long as $\operatorname{Rank}\{\mathbf{H}(k)\}=\operatorname{Rank}\left\{\mathbf{P}_{\mathbf{S}(k)}^{\perp} \mathbf{H}(k)\right\} .{ }^{2}$ Notice that the MMSE detector approaches the GML detector only when the interference-nulled correlation matrix, $\mathbf{H}^{*}(k) \mathbf{P}_{\mathbf{S}(k)}^{\perp} \mathbf{H}(k)$, is a scalar multiple of the identity matrix.

Proof: See Appendix A.

The detector suggested in Theorem IV.1 appears to be original and we call it the multipulse decorrelating (MD) detector. Using the results of [12], we find that the MD detector may be derived by maximizing the likelihood function

$$
f(\mathbf{y})=\frac{1}{\left(\sigma^{2} \pi\right)^{N}} \exp \left\{-\frac{1}{\sigma^{2}}\|\mathbf{y}-\mathbf{H}(k) \mathbf{D}(k) \mathbf{b}(k)-\mathbf{S}(k) \beta\|^{2}\right\}_{(9)}
$$

jointly over $\mathrm{D}(k) \mathrm{b}(k)$ and $\beta$, and choosing $m$ as the entry of $\mathbf{D}(\widehat{k) \mathbf{b}}(k)$ of largest magnitude. It is, perhaps, worth noting that the MD detector estimates the signal $\mathbf{H}(k) \mathbf{D}(k) \mathbf{b}(k)$ before imposing the a priori knowledge that this term is of the form $\sqrt{E_{k}} e^{j \theta_{m}(k)} \mathbf{h}_{m}(k)$, whereas the GML detector imposes this constraint from the outset.

\section{Asymptotic Performance ANAlysis}

In this section, we analyze the performance of the MD detector. As this performance characterizes the MMSE detector asymptotically, it is useful in the investigation of both detectors. We can build asymptotically tight bounds on the probability of error, $P^{\mathrm{MD}}$, for the MD detector via

$$
\max _{l \neq m}-P^{\mathrm{MD}}(m, l) \leq P^{\mathrm{MD}} \leq \frac{1}{M} \sum_{m=1}^{M} \sum_{\substack{l=1 \\ l \neq m}}^{M} P^{\mathrm{MD}}(m, l)
$$

where $P^{\mathrm{MD}}(m, l)$ is the probability that the $l$ th statistic in $(8)$ is greater then the $m$ th statistic when signal $m$ is transmitted. The upper and lower bounds are asymptotically coincident on the AWGN channel and so we will concentrate on the lower bound.

\footnotetext{
${ }^{2}$ This condition implies that $\mathbf{H}(k)$ and $\mathbf{S}(k)$ are linearly independent, i.e., that if $\operatorname{Rank}\{\mathbf{H}(k)\}=r$ and $\operatorname{Rank}\{\mathbf{S}(k)\}=p$, then $\operatorname{Rank}\{[\mathbf{H}(k) \mathbf{S}(k)]\}=$ $p+r$. This may be assumed without loss of generality since otherwise user $k$ is wasting power by communicating along a coordinate vector lying completely in the span of the interference. This condition does not require the matrix $\mathbf{H}(k)$ to be full rank (we can have $r<M$ ).
} 
Each term, $P^{\mathrm{MD}}(m, l)$, is found from

$$
\begin{aligned}
& P^{\mathrm{MD}}(m, l) \\
&=\operatorname{Prob}[\mid\left.\left\{\left(\mathbf{G}^{*}(k) \mathbf{G}(k)\right)^{+} \mathbf{G}_{m}^{*}(k) \mathbf{y}\right\}_{m}\right|^{2} \\
&\left.<\left|\left\{\left(\mathbf{G}^{*}(k) \mathbf{G}(k)\right)^{+} \mathbf{G}^{*}(k) \mathbf{y}\right\}_{l}\right|^{2} \mid \text { Hyp. } m\right] \\
&=\operatorname{Prob}\left[\mathbf{y}^{*} \mathbf{G}\left(\mathbf{G}^{*} \mathbf{G}\right)^{+}\left(\mathbf{e}_{m} \mathbf{e}_{m}^{T}-\mathbf{e}_{l} \mathbf{e}_{l}^{T}\right)\right. \\
&\left.\cdot\left(\mathbf{G}^{*} \mathbf{G}\right)^{+} \mathbf{G}^{*} \mathbf{y}<0 \mid \text { Hyp. } m\right]
\end{aligned}
$$

where $\mathbf{e}_{m}$ is the $m$ th column of the identity matrix, $\mathbf{G}=\mathbf{P}_{\mathbf{S}(k)}^{\perp} \mathbf{H}(k)$, and we have dropped the dependency on $k$.

Let us form the eigendecomposition $\mathbf{G}^{*} \mathbf{G}=\mathbf{U} \boldsymbol{\Gamma} \mathbf{U}^{*}$, where $\boldsymbol{\Gamma} \in \mathbb{R}^{r \times r}$ and $r=\operatorname{Rank}\{\mathbf{G}\}$. Define

$$
\begin{aligned}
\mathbf{w} & =\frac{e^{-j \theta_{m}}}{\sigma} \mathbf{U}^{*}\left(\mathbf{G}^{*} \mathbf{G}\right)^{+/ 2} \mathbf{G}^{*} \mathbf{y} \text { and } \\
\mathbf{Q} & =\mathbf{U}^{*}\left(\mathbf{G}^{*} \mathbf{G}\right)^{+/ 2}\left(\mathbf{e}_{m} \mathbf{e}_{m}^{T}-\mathbf{e}_{\mathbf{e}} \mathbf{e}_{l}^{T}\right)\left(\mathbf{G}^{*} \mathbf{G}\right)^{+/ 2} \mathbf{U} \\
& =\left[\begin{array}{ll}
\mathbf{v}_{1} & \mathbf{v}_{2}
\end{array}\right]\left[\begin{array}{cc}
\lambda_{1} & 0 \\
0 & \lambda_{2}
\end{array}\right]\left[\begin{array}{c}
\mathbf{v}_{1}^{*} \\
\mathbf{v}_{2}^{*}
\end{array}\right]
\end{aligned}
$$

then we have

$$
P^{\mathrm{MD}}(m, l)=\operatorname{Prob}\left[\mathbf{w}^{*} \mathbf{Q w}<0 \mid H_{m}\right] .
$$

We require that the matrix $\mathbf{Q}$ have rank 2, if this condition is not met then either $P^{\mathrm{MD}}(m, l)=1$ or $P^{\mathrm{MD}}(l, m)=1$, resulting in a probability of error which approaches a constant as the SNR grows.

The vector $\mathbf{w}$ is complex normal with correlation matrix $E\left[\mathbf{w w}^{*}\right]=\mathbf{I}$ and mean $E[\mathbf{w}]=\mathbf{f}=(1 / \sigma) \mathbf{U}^{*}\left(\mathbf{G}^{*} \mathbf{G}\right)^{1 / 2} \mathbf{e}_{m}$ under hypothesis $m$. We may use the results of [13, Appendix B] and of [14, Appendix B] to find

$$
P^{\mathrm{MD}}(m, l)=Q_{1}(a, b)-\frac{\omega_{2} / \omega_{1}}{1+\omega_{2} / \omega_{1}} e^{-(1 / 2)\left(a^{2}+b^{2}\right)} I_{0}(a b)
$$

where $Q_{1}(\cdot)$ is the Marcum Q-function, $I_{0}(\cdot)$ is the zeroth-order modified Bessel function, and we have defined

$$
\begin{aligned}
a & =2 \sqrt{\frac{2 \omega_{1}^{2} \omega_{2}\left(\alpha_{1} \omega_{2}-\alpha_{2}\right)}{\left(\omega_{1}+\omega_{2}\right)^{2}}} \quad b=2 \sqrt{\frac{2 \omega_{1} \omega_{2}^{2}\left(\alpha_{1} \omega_{1}+\alpha_{2}\right)}{\left(\omega_{1}+\omega_{2}\right)^{2}}} \\
\omega_{1} & =\frac{1}{4 \lambda_{1}} \quad \omega_{2}=-\frac{1}{4 \lambda_{2}} \\
\alpha_{1} & =-4 \lambda_{1} \lambda_{2}\left(\mathbf{v}_{1} \mathbf{v}_{1}^{*}+\mathbf{v}_{2} \mathbf{v}_{2}^{*}\right) \quad \alpha_{2}=\mathbf{f}^{*} \mathbf{Q f} .
\end{aligned}
$$

We are interested in the performance as the noise power becomes small and so we consider the asymptotic approximation to $P^{\mathrm{MD}}(m, l)$ found from the relations in [13, Appendix A]

$$
P^{\mathrm{MD}}(m, l) \sim e^{-(1 / 2)(b-a)^{2}}
$$

for small values of $\sigma^{2}$. This implies that we should study the distance measure $(b-a)^{2}$. This is done in Appendix B of this letter and we find

$$
(b-a)^{2} \sim \frac{2}{\sigma^{2}\left(\left(\mathbf{G}^{*} \mathbf{G}\right)_{m, m}^{+}+\left(\mathbf{G}^{*} \mathbf{G}\right)_{l, l}^{+}+2\left|(\mathbf{G} * \mathbf{G})_{m, l}^{+}\right|\right)} .
$$

Since the performance is asymptotically dominated by the largest pairwise error, we find that as the noise power vanishes we have (18), shown at the bottom of the page.

\section{Multiuser Performance Measures}

In this section, we use our asymptotic expression for the probability of error to derive the asymptotic multiuser efficiency (AME) and the near-far resistance of the MMSE and MD detectors. The AME of the $k$ th user is defined by [9]

$$
\eta(k)=\sup \left\{0 \leq r \leq 1, \lim _{\sigma^{2} \rightarrow 0} \frac{P_{\phi}\left(\sigma^{2}\right)}{P_{S U}\left(\sigma^{2} / r\right)}<\infty\right\}
$$

where $P_{\phi}\left(\sigma^{2}\right)$ is the probability of error for the $k$ th user employing the MMSE (or MD) detector $\phi$ with additive white Gaussian noise (AWGN) power $\sigma^{2}$, and $P_{S U}\left(\sigma^{2} / r\right)$ is the probability of error for the MMSE (MD) detector in the absence of interfering users $(\mathbf{S}=\mathbf{0})$ with effective noise power $\sigma^{2} / r$.

Using the asymptotically tight expressions for $P_{\phi}\left(\sigma^{2}\right)$ and $P_{S U}\left(\sigma^{2} r^{-1}\right)$ given in (18) (in the latter case we simply set $\mathbf{P}_{\mathbf{S}}^{\perp}=\mathbf{I}$ ), we find (20), shown at the bottom of the page. The near far resistance of the detector is defined as the infimum of the AME over the possible realizations of the interfering users' powers. As the MMSE acts asymptotically to null the MAI, we find that the near-far resistance is simply the AME given in (20).

\section{COMPARISON WITH THE GML RULE}

It is interesting to compare the error expression in (18) with the asymptotic expression for the probability of error of the GML detector. This latter quantity is known to be [3]-[5]

$$
\begin{aligned}
P^{\mathrm{GML}} \sim \exp & \left\{\frac{-E_{k}}{2 \sigma^{2}} \min _{m \neq l}\left\|\mathbf{P}_{\mathbf{S}}^{\perp} \mathbf{h}_{m}\right\|^{2}\right. \\
& \left.\times\left(1-\frac{\left|\mathbf{h}_{m}^{*} \mathbf{P}_{\mathbf{S}}^{\perp} \mathbf{h}_{l}\right|}{\left\|\mathbf{P}_{\mathbf{S}}^{\perp} \mathbf{h}_{m}\right\|\left\|\mathbf{P}_{\mathbf{S}}^{\perp} \mathbf{h}_{l}\right\|}\right)\right\} .
\end{aligned}
$$

$$
P^{\mathrm{MD}} \sim \exp \left\{\min _{l \neq m} \frac{-1}{\sigma^{2}\left(\left(\mathbf{H}^{*} \mathbf{P}_{\mathbf{S}}^{\perp} \mathbf{H}\right)_{m, m}^{+}+\left(\mathbf{H}^{*} \mathbf{P}_{\mathbf{S}}^{\perp} \mathbf{H}\right)_{l, l}^{+}+2\left|\left(\mathbf{H}^{*} \mathbf{P}_{\mathbf{S}}^{\perp} \mathbf{H}\right)_{m, l}^{+}\right|\right)}\right\}
$$

$$
\eta(k)=\frac{\min _{l \neq m}\left(\mathbf{H}^{*} \mathbf{H}\right)_{l, l}^{+}+\left(\mathbf{H}^{*} \mathbf{H}\right)_{m, m}^{+}+2\left|\left(\mathbf{H}^{*} \mathbf{H}\right)_{l, m}^{+}\right|}{\min _{n \neq p}\left(\mathbf{H}^{*} \mathbf{P}_{\mathbf{S}}^{\perp} \mathbf{H}\right)_{n, n}^{+}+\left(\mathbf{H}^{*} \mathbf{P}_{\mathbf{S}}^{\perp} \mathbf{H}\right)_{p, p}^{+}+2\left|\left(\mathbf{H}^{*} \mathbf{P}_{\mathbf{S}}^{\perp} \mathbf{H}\right)_{n, p}^{+}\right|}
$$




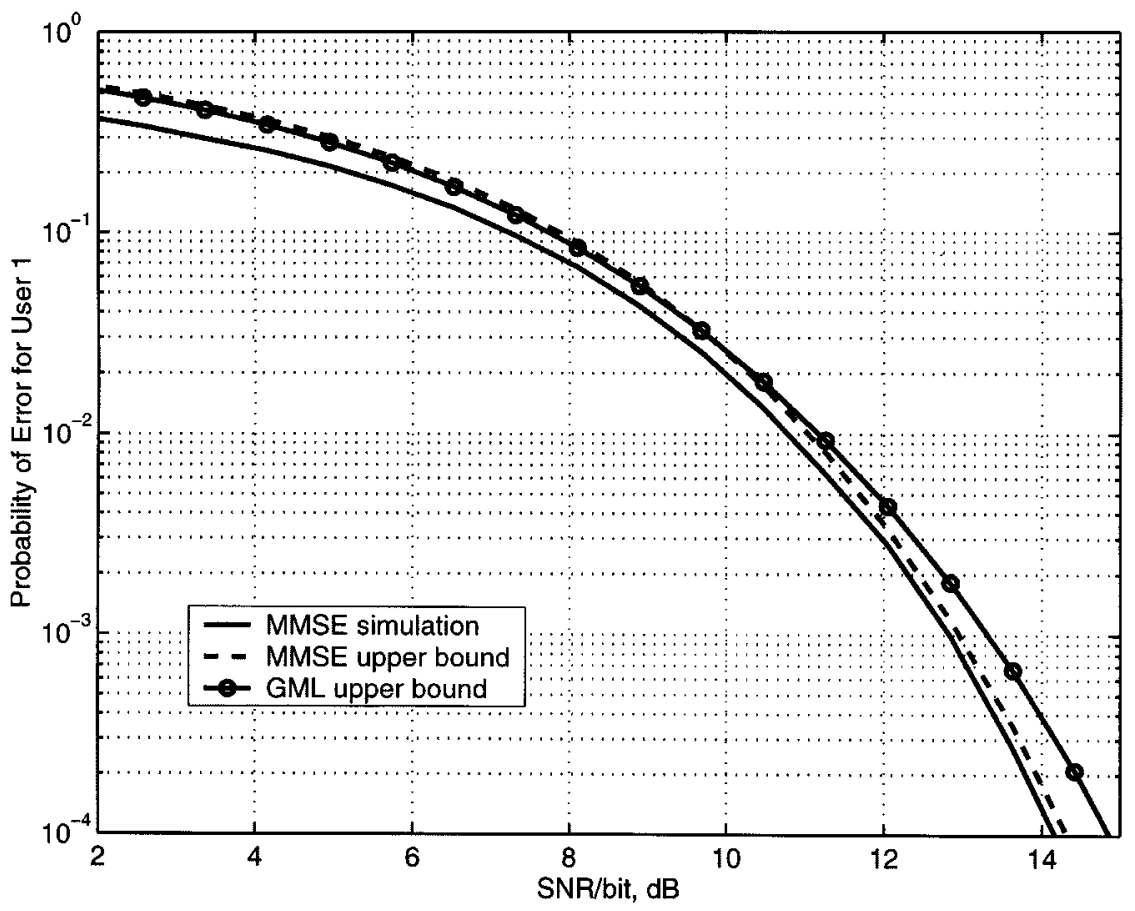

Fig. 1. Results for the first example of Section VIII. Plotted are the probability of symbol error for the MMSE detector (solid) along with the asymptotic union bound of (10) (dashed) and the union bound on the GML detector (circles).

It is clear that the expression in (18) and (21) are not generally equal. They are equal for the case of orthogonal signal with respect to $\mathbf{P}_{\mathbf{S}}^{\perp}$, i.e., $\mathbf{H}^{*} \mathbf{P}_{\mathbf{S}}^{\perp} \mathbf{H}=\nu \mathbf{I}$, which is clear from the definitions of the two tests. In general there is no clear reason to choose the MMSE (MD) over the GML detector, at least in terms of asymptotic performance. For the case of binary signaling $(M=2)$, however, we show in the following section that asymptotically the MMSE (MD) detector is superior.

\section{A. MMSE Detector is Superior for Binary Signaling}

For binary signaling we let $\mathbf{G}=\mathbf{P} \stackrel{\perp}{\perp} \mathbf{H}=\left[\mathbf{g}_{1}, \mathbf{g}_{2}\right]$. Then the MMSE (MD) detector has the asymptotic probability of error $\exp \left\{-E_{k} \alpha_{M} /\left(2 \sigma^{2}\right)\right\}$ with

$$
\begin{aligned}
\alpha_{M} & =\frac{2}{\left(\mathbf{G}^{*} \mathbf{G}\right)_{1,1}^{-1}+\left(\mathbf{G}^{*} \mathbf{G}\right)_{2,2}^{-1}+2\left|\left(\mathbf{G}^{*} \mathbf{G}\right)_{1,2}^{-1}\right|} \\
& =\frac{2}{\frac{1}{\mathbf{g}_{1}^{*} \mathbf{P}_{\mathbf{g}_{2}}^{\perp} \mathbf{g}_{1}}+\frac{1}{\mathbf{g}_{2}^{*} \mathbf{P}_{\mathbf{g}_{1}}^{\perp} \mathbf{g}_{2}}+2 \frac{\left|\mathbf{g}_{1}^{*} \mathbf{P}_{\mathbf{g}_{2}}^{\perp} \mathbf{P}_{\mathbf{g}_{1}}^{\perp} \mathbf{g}_{2}\right|}{\left(\mathbf{g}_{1}^{*} \mathbf{P}_{\mathbf{g}_{2}}^{\perp} \mathbf{g}_{1}\right)\left(\mathbf{g}_{2}^{*} \mathbf{P}_{\mathbf{g}_{1}}^{\perp} \mathbf{g}_{2}\right)}} \\
& =\frac{2\left(A B-|\beta|^{2}\right)}{A+B+2|\beta|},
\end{aligned}
$$

where $A=\left\|\mathbf{g}_{1}\right\|^{2}, B=\left\|\mathbf{g}_{2}\right\|^{2}$, and $\beta=\mathbf{g}_{1}^{*} \mathbf{g}_{2}$. We have assumed that $\mathbf{G}$ is invertible, since otherwise both the MD and the GML test fail.

In light of (21) we see that asymptotically, the GML rule has probability of error $\exp \left\{-E_{k} \alpha_{G} /\left(2 \sigma^{2}\right)\right\}$. The exponential parameter $\alpha_{G}$ is given by

$$
\alpha_{G}=B\left(1-\frac{|\beta|}{\sqrt{A B}}\right)
$$

assuming without loss of generality that $A \geq B$ (if $B>A$ we simply switch $A$ and $B$ ).

We are interested in the ratio $\alpha_{M} / \alpha_{G}$, noticing that if this ratio is greater then one we have the MMSE (MD) outperforming the GML detector (asymptotically). Assuming that $A \geq B$ we have

$$
\begin{aligned}
\frac{\alpha_{M}}{\alpha_{G}} & =\frac{2 A+2|\beta| \sqrt{A / B}}{A+B+2|\beta|} \\
& \geq \frac{2 A+2|\beta| \sqrt{A / B}}{2 A+2|\beta|} \quad(\text { since } A+B \leq 2 A) \\
& \geq 1 \quad(\text { since } A / B \geq 1) .
\end{aligned}
$$

The same argument works for the case of $B \geq A$ and we conclude that the MMSE (MD) detector is superior to the GML detector for large SNRs. Notice that equality is achieved when $A=B$.

This appears to be the most general statement that can be made about the asymptotic performance difference between the two detectors. For every value of $M$ greater then two that we have considered, we have found signal sets for which $\alpha_{G}>$ $\alpha_{M}$.

\section{A NUMERICAL EXAMPLE}

We consider a noncoherent channel with $K=3$ users, each employing $M=3$ signals. The signals were taken to be length $N=31$ Gold codes (user one used codes 5-7, user two used codes 8-10, etc.), normalized to have unit norm. The user energies were chosen to be $E_{1}=1, E_{2}=5$, and $E_{3}=5$. The probability of error for the MMSE detector was estimated by Monte-Carlo simulation. The results are shown in Fig. 1 along with the union upper bound on the MD detector of (10). Notice 


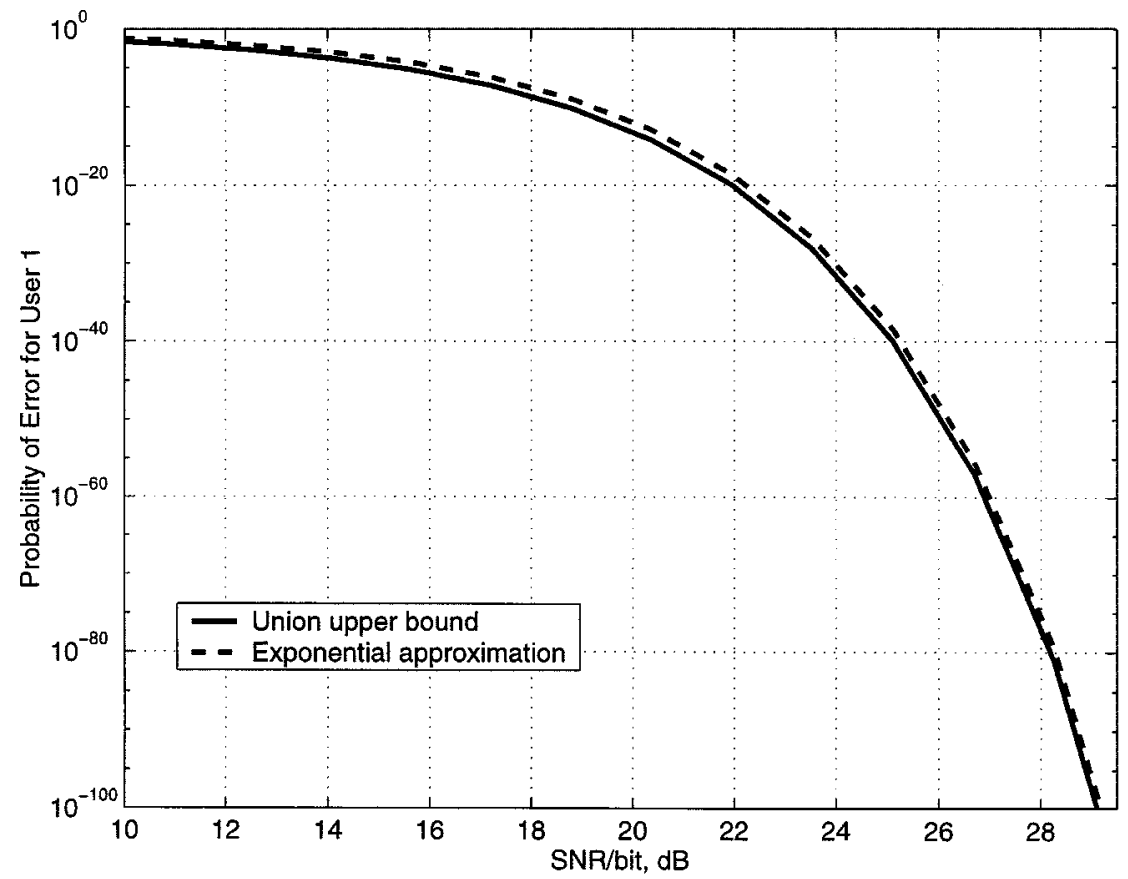

Fig. 2. Comparison of the union upper bound given in (10) with the exponential approximation of (18) for the example of Section VIII.

that fit between the MMSE performance and the bound on the MD detector appears quite good for this problem, even at relatively low SNRs. For comparison, we have also plotted the union bound for the GML detector (see, e.g., [5]). For this example, $\alpha_{M}=0.3445$ and $\alpha_{G}=0.2674$, and the MMSE detector outperforms the GML, as expected. The exponential bound of (18) is plotted against the union bound of (10) in Fig. 2. We see that the two bounds are asymptotically coincident, as predicted.

\section{CONCLUSIONS}

In this letter we have studied the MMSE, MD, and GML detectors for noncoherent NMM. It was observed that the former two detectors are asymptotically superior to the latter for binary signaling, a result which does not appear to generalize to larger cardinality signal constellations. The AME and near-far resistance were derived for the MMSE and MD detectors through a large SNR approximation to the probability of error of the detectors.

The MMSE detector requires knowledge of the users' energy levels and of the interfering users' signal vectors. These requirement can be lifted by replacing the measurement correlation matrix by an estimate based on several observations. Blind detection along these lines is considered in [6].

APPENDIX A

PROOF OF THEOREM IV.1

Rewrite $\mathbf{R}$ as $\mathbf{R}=\left(E_{k} / M\right) \mathbf{H H}^{*}+\mathbf{B}$, with $\mathbf{B}=(1 / M) \sum_{l \neq k} E_{l} \mathbf{H}(l) \mathbf{H}^{*}(l)+\sigma^{2} \mathbf{I}$ and $E_{k}=E\left[|\mu(k)|^{2}\right.$.
Application of the Woodbury identity yields

$$
\mathbf{H}^{*} \mathbf{R}^{-1} \mathbf{y}=\frac{M}{E_{k}}\left(\frac{M}{E_{k}} \mathbf{I}+\mathbf{H}^{*} \mathbf{B}^{-1} \mathbf{H}\right)^{-1} \mathbf{H}^{*} \mathbf{B}^{-1} \mathbf{y} .
$$

Let $\mathbf{B}$ have the eigendecomposition

$$
\mathbf{B}=\left[\mathbf{U}_{S} \mathbf{U}_{N}\right]\left[\begin{array}{cc}
\boldsymbol{\Sigma}_{S}+\sigma^{2} \mathbf{I} & \mathbf{0} \\
\mathbf{0} & \sigma^{2} \mathbf{I}
\end{array}\right]\left[\begin{array}{c}
\mathbf{U}_{S}^{*} \\
\mathbf{U}_{N}^{*}
\end{array}\right]
$$

Then we have $\sigma^{2} \mathbf{B}^{-1}=\sigma^{2} \mathbf{U}_{S}\left(\boldsymbol{\Sigma}_{S}+\sigma^{2} \mathbf{I}\right)^{-1} \mathbf{U}_{S}^{*}+\mathbf{P}_{\mathbf{S}(k)}^{\perp}$ where $\mathbf{P}_{\mathbf{S}(k)}=\mathbf{U}_{S} \mathbf{U}_{S}^{*}$ and $\mathbf{P}_{\mathbf{S}(k)}^{\perp}=\mathbf{U}_{N} \mathbf{U}_{N}^{*}$. Notice that $\sigma^{2} \mathbf{B}^{-1} \rightarrow$ $\mathbf{P}_{\mathbf{S}(k))}^{\perp}$ as $\sigma^{2}$ grows small since $\sigma^{2} \mathbf{U}_{S}\left(\boldsymbol{\Sigma}_{S}+\sigma^{2} \mathbf{I}\right)^{-1} \mathbf{U}_{S}^{*} \rightarrow \mathbf{0}$.

We next perform the singular value decomposition $\mathbf{H}^{*} \mathbf{P}_{\mathbf{S}(k)}^{\perp}=\mathbf{V} \boldsymbol{\Lambda} \mathbf{U}^{*}$, where $\boldsymbol{\Lambda}$ is a full rank diagonal


tary. It follows that $\mathbf{W}^{*} \mathbf{H}^{*}=\mathbf{0}$ since $\langle\mathbf{W}\rangle$ is orthogonal to $\left\langle\mathbf{H}^{*} \mathbf{P}_{\mathbf{S}(k)}^{\perp}\right\rangle$ and $\left\langle\mathbf{H}^{*}\right\rangle=\left\langle\mathbf{H}^{*} \mathbf{P}_{\mathbf{S}(k)}^{\perp}\right\rangle$ by our assumption on the dimension of each subspace. Now consider the matrix

$$
\begin{aligned}
\frac{E_{k}}{M} & \mathbf{H}^{*} \mathbf{R}^{-1} \\
= & \left(\frac{M \sigma^{2}}{E_{k}} \mathbf{I}+\mathbf{H}^{*} \sigma^{2} \mathbf{B}^{-1} \mathbf{H}\right)^{-1} \mathbf{H}^{*} \sigma^{2} \mathbf{B}^{-1} \\
= & \left(\frac{M \sigma^{2}}{E_{k}} \mathbf{I}+\mathbf{H}^{*}\left(\sigma^{2} \mathbf{U}_{S}\left(\Sigma_{\mathbf{S}}+\sigma^{2} \mathbf{I}\right)^{-1} \mathbf{U}_{\mathbf{S}}^{*}+\mathbf{P}_{\mathbf{S}(\mathbf{k})}^{\perp}\right) \mathbf{H}\right)^{-1} \\
& \times \mathbf{H}^{*}\left(\sigma^{2} \mathbf{U}_{S}\left(\Sigma_{\mathbf{S}}+\sigma^{\mathbf{2}} \mathbf{I}\right)^{-1} \mathbf{U}_{\mathbf{S}}^{*}+\mathbf{P}_{\mathbf{S}(\mathbf{k})}^{\perp}\right) \\
= & \left(\frac{M \sigma^{2}}{E_{k}} \mathbf{I}+\sigma^{2} \mathbf{H}^{*} \mathbf{F} \mathbf{H}+\mathbf{H}^{*} \mathbf{P}_{\mathbf{S}(k)}^{\perp} \mathbf{H}\right)^{-1} \\
& \times \mathbf{H}^{*}\left(\sigma^{2} \mathbf{F}+\mathbf{P}_{\mathbf{S}(k)}^{\perp}\right)
\end{aligned}
$$


where $\mathbf{F}=\mathbf{U}_{S}\left(\boldsymbol{\Sigma}_{\mathbf{S}}+\sigma^{\mathbf{2}} \mathbf{I}\right)^{-\mathbf{1}} \mathbf{U}_{\mathbf{S}}^{*}$. This result may be resolved onto the basis $\left[\begin{array}{ll}\mathbf{V} & \mathbf{W}\end{array}\right]$ as follows:

$$
\begin{aligned}
& \frac{E_{k}}{M} \mathbf{H}^{*} \mathbf{R}^{-1} \\
&= {[\mathbf{V} \quad \mathbf{W}] } \\
& \times\left[\begin{array}{cc}
\frac{M \sigma^{2}}{E_{k}} \mathbf{I}+\sigma^{2} \mathbf{V}^{*} \mathbf{H}^{*} \mathbf{F H V}+\mathbf{\Lambda}^{2} & \mathbf{0} \\
\mathbf{0} & \frac{M \sigma^{2}}{E_{k}} \mathbf{I}
\end{array}\right]^{-1} \\
& \times\left[\begin{array}{l}
\mathbf{V}^{*} \\
\mathbf{W}^{*}
\end{array}\right] \mathbf{H}^{*}\left(\sigma^{2} \mathbf{F}+\mathbf{P}_{\mathbf{S}(k)}^{\perp}\right) \\
&= \mathbf{V}\left(\frac{M \sigma^{2}}{E_{k}} \mathbf{I}+\sigma^{2} \mathbf{V}^{*} \mathbf{H}^{*} \mathbf{F H V}+\mathbf{\Lambda}^{2}\right)^{-1} \\
& \times \mathbf{V}^{*} \mathbf{H}^{*}\left(\sigma^{2} \mathbf{F}+\mathbf{P}_{\mathbf{S}(k)}^{\perp}\right)
\end{aligned}
$$

since $\mathbf{W}^{*} \mathbf{H}^{*}=\mathbf{0}$. Now, as $\sigma^{2} \rightarrow 0$, we find

$$
\begin{aligned}
\frac{E_{k}}{M} \mathbf{H}^{*} \mathbf{R}^{-1} & \rightarrow \mathbf{V} \Lambda^{-2} \mathbf{V}^{*} \mathbf{H}^{*} \mathbf{P}_{\mathbf{S}(k)}^{\perp} \\
& =\mathbf{V} \Lambda^{-1} \mathbf{U}^{*} \\
& =\left(\mathbf{P}_{\mathbf{S}(k)}^{\perp} \mathbf{H}\right)^{+} \\
& =\left(\mathbf{H}^{*} \mathbf{P}_{\mathbf{S}(k)}^{\perp} \mathbf{H}\right)^{+} \mathbf{H}^{*} \mathbf{P}_{\mathbf{S}(k)}^{\perp}
\end{aligned}
$$

where we have used the standard construction of the pseudoinverse of $\mathbf{P}_{\mathbf{S}(k)}^{\perp} \mathbf{H}$ in terms of its singular value decomposition. The term $\left(E_{k} / M\right)$ is hypothesis independent and may be dropped.

\section{APPENDIX B}

DERIVATION OF THE PAIRWISE ERROR EXPONENTS

After straightforward calculation, we find

$$
\begin{aligned}
(b- & a)^{2} \\
= & \frac{2}{\lambda_{1}-\lambda_{2}} \\
& \times\left(\lambda_{1} \mathbf{f}^{*} \mathbf{P}_{1} \mathbf{f}-\lambda_{2} \mathbf{f}^{*} \mathbf{P}_{2} \mathbf{f}-2 \sqrt{-\lambda_{1} \lambda_{2}\left(\mathbf{f}^{*} \mathbf{P}_{1} \mathbf{f}\right)\left(\mathbf{f}^{*} \mathbf{P}_{2} \mathbf{f}\right)}\right)
\end{aligned}
$$

where $\mathbf{P}_{1}=\mathbf{v}_{1} \mathbf{v}_{1}^{*}$ and $\mathbf{P}_{2}=\mathbf{v}_{2} \mathbf{v}_{2}^{*}$. To gain insight into this expression we need to solve for the eigenvalues and eigenvectors of the matrix $\mathbf{Q}$. Let $\mathbf{a}_{1}$ and $\mathbf{a}_{2}$ be the $m$ th and $l$ th column of $\mathbf{U}^{*}\left(\mathbf{G}^{*} \mathbf{G}\right)^{+/ 2}$, respectively, so that $\mathbf{Q}=\mathbf{a}_{1} \mathbf{a}_{1}^{*}-\mathbf{a}_{2} \mathbf{a}_{2}^{*}$. Let us define the Gram-Schmidt vectors

$$
\mathbf{z}_{1}=\frac{\mathbf{a}_{1}}{\left\|\mathbf{a}_{1}\right\|} \quad \text { and } \quad \mathbf{z}_{2}=\frac{\left(\mathbf{I}-\mathbf{z}_{1} \mathbf{z}_{1}^{*}\right) \mathbf{a}_{2}}{\left\|\left(\mathbf{I}-\mathbf{z}_{1} \mathbf{z}_{1}^{*}\right) \mathbf{a}_{2}\right\|} .
$$

Then for $\mathbf{Z}=\left[\mathbf{z}_{1}, \mathbf{z}_{2}\right]$, we have $\mathbf{Z} \mathbf{Z}^{*} \mathbf{Q} \mathbf{Z} \mathbf{Z}^{*}=\mathbf{Q}$ and consequently the nonzero eigenvalues of the matrix $\mathbf{T}=\mathbf{Z}^{*} \mathrm{QZ}$ are equal to the those of $\mathbf{Q}$. We find the matrix $\mathbf{T}$ to be

$$
\mathbf{T}=\mathbf{Z Q Z}^{*}=\left[\begin{array}{cc}
\gamma_{1}-\frac{|\rho|^{2}}{\gamma_{1}} & -\rho \sqrt{\frac{\gamma_{2}}{\gamma_{1}}-\frac{|\rho|^{2}}{\gamma_{1}^{2}}} \\
-\bar{\rho} \sqrt{\frac{\gamma_{2}}{\gamma_{1}}-\frac{|\rho|^{2}}{\gamma_{1}^{2}}} & -\left(\gamma_{2}-\frac{|\rho|^{2}}{\gamma_{1}}\right)
\end{array}\right]
$$

where

$$
\begin{aligned}
\gamma_{1} & =\mathbf{a}_{1}^{*} \mathbf{a}_{1}=\left(\mathbf{G}^{*} \mathbf{G}\right)_{m, m}^{+} \quad \gamma_{2}=\mathbf{a}_{2}^{*} \mathbf{a}_{2}=\left(\mathbf{G}^{*} \mathbf{G}\right)_{l, l}^{+} \\
\rho & =\mathbf{a}_{1}^{*} \mathbf{a}_{2}=\left(\mathbf{G}^{*} \mathbf{G}\right)_{m, l}^{+} .
\end{aligned}
$$
and

The eigenvalues of $\mathbf{Q}$ are found to be

$$
\lambda_{k}=\frac{1}{2}\left(\gamma_{1}-\gamma_{2} \pm \sqrt{\left(\gamma_{1}+\gamma_{2}\right)^{2}-4|\rho|^{2}}\right) .
$$

To find the eigenvectors of $\mathbf{Q}$ we first notice that if $\mathbf{T}$ has the spectral decomposition $\mathbf{T}=\mathbf{W} \Lambda \mathbf{W}^{*}$, then we may decompose $\mathbf{Q}$ as

$$
\mathbf{Q}=\mathbf{V} \Lambda \mathbf{V}^{*}=\mathbf{Z} \mathbf{T} \mathbf{Z}^{*}=\mathbf{Z W} \Lambda \mathbf{W}^{*} \mathbf{Z}^{*}
$$

We see that given $\mathbf{W}$, we may find the eigenvectors of $\mathbf{Q}$ through the relation $\mathbf{V}=\mathbf{Z W}$. The eigenvectors of $\mathbf{T}$ are given by

$$
\mathbf{w}_{k}=\frac{1}{\sqrt{1+\frac{\left|t_{2}\right|^{2}}{\left(t_{1}-\lambda_{k}\right)^{2}}}}\left[-\frac{t_{2}}{t_{1}-\lambda_{k}}\right]
$$

where $t_{1}=\{\mathbf{T}\}_{1,1}$ and $t_{2}=\{\mathbf{T}\}_{1,2}$.

To resolve the quadratic forms $\mathbf{f}^{*} \mathbf{P}_{k} \mathbf{f}$ that appear in (29), we notice that $\mathbf{f}^{*} \mathbf{z}_{1}=\left(1 / \sigma \sqrt{\gamma_{1}}\right)$ and $\mathbf{f}^{*} \mathbf{z}_{2}=$ $-\left(\rho / \sigma \gamma_{1} \sqrt{\gamma_{2}-|\rho|^{2} / \gamma_{1}}\right)$ by virtue of the definitions of $\mathbf{f}$ and the $\mathbf{a}_{k}$ 's. This allows us to expand the quadratic forms as

$$
\begin{aligned}
\mathbf{f}^{*} \mathbf{P}_{k} \mathbf{f} & =\mathbf{f}^{*} \mathbf{Z} \mathbf{w}_{k} \mathbf{w}_{k}^{*} \mathbf{Z}^{*} \mathbf{f} \\
& =\frac{1}{\sigma^{2}\left(\left|t_{2}\right|^{2}+\left(t_{1}-\lambda_{k}\right)^{2}\right)}\left|\frac{t_{2}}{\sqrt{\gamma_{1}}}+\frac{\rho\left(t_{1}-\lambda_{k}\right)}{\gamma_{1} \sqrt{\gamma_{2}-|\rho|^{2} / \gamma_{1}}}\right|^{2} .
\end{aligned}
$$

Substituting in for the values of $t_{1}$ and $t_{2}$, we find the two basic quantities appearing in (29) to be given by (38), shown at the top of the page. Substituting these expressions into (29) and simplifying the resulting expression yields

$$
(b-a)^{2}=\frac{2}{\sigma^{2}\left(\gamma_{1}+\gamma_{2}+2|\rho|\right)} .
$$

$$
\begin{aligned}
\lambda_{k} \mathbf{f}^{*} \mathbf{P}_{k} \mathbf{f}= & \frac{1}{\sigma^{2}}\left(\frac{\left(\gamma_{1}-\gamma_{2} \pm \sqrt{\left(\gamma_{1}+\gamma_{2}\right)^{2}-4|\rho|^{2}}\right)}{\left[\left(\gamma_{1}+\gamma_{2}\right)^{2}-4|\rho|^{2}\right] \mp\left(\gamma_{1}+\gamma_{2}-2|\rho|^{2} / \gamma_{1}\right) \sqrt{\left(\gamma_{1}+\gamma_{2}\right)^{2}-4|\rho|^{2}}}\right) \\
& \cdot\left|\frac{\rho}{2 \gamma_{1} \sqrt{\gamma_{2}-|\rho|^{2} / \gamma_{1}}}\left(\gamma_{1}-\gamma_{2} \pm \sqrt{\left(\gamma_{1}+\gamma_{2}\right)^{2}-4|\rho|^{2}}\right)\right|^{2} .
\end{aligned}
$$




\section{REFERENCES}

[1] A. Russ, "Noncoherent detection for nonlinear binary modulation in Gaussian CDMA channels," M.S. thesis, Friedrich Alexander Universitaet, Erlangen-Nuremberg, Germany, Sept. 1996.

[2] M. L. McCloud, L. L. Scharf, and L. T. McWhorter, "Subspace coherence for detection in multiuser additive noise channels," in Proc. SPAWC '97, Paris, France, Apr. 1997, pp. 225-228.

[3] M. K. Varanasi and A. Russ, "Noncoherent decorrelative multiuser detection for nonlinear nonorthogonal modulation," in Proc. I.C.C.'97, Montreal, Canada, June 1997.

[4] M. L. McCloud and L. L. Scharf, "Generalized likelihood detection on multiple access channels," in Proc. Asilomar '97, Monterey, CA, Nov. 1997.

[5] M. K. Varanasi and A. Russ, "Noncoherent decorrelative detection for nonorthogonal multipulse modulation over the multiuser Gaussian channel," IEEE Trans. Commun., vol. 46, pp. 1675-1684, Dec. 1998.

[6] M. L. McCloud and L. L. Scharf, "Interference estimation with applications to blind multiple access communication over fading channels," IEEE Trans. Inform. Theory, vol. 46, pp. 947-961, May 2000.
[7] X. Wang and H. V. Poor, "Blind multiuser detection: A subspace approach," IEEE Trans. Inform. Theory, vol. 44, pp. 677-689, Mar. 1998

[8] U. Madhow and M. L. Honig, "MMSE interference suppression for direct-sequence spread-spectrum CDMA," IEEE Trans. Commun., vol. 42, pp. 3178-3188, Dec. 1994.

[9] R. Lupas and S. Verdu, "Linear multiuser detectors for synchronous code-division multiple-access channels," IEEE Trans. Inform. Theory, vol. 35, pp. 123-136, Jan. 1989.

[10] M. K. Varanasi and D. Das, "Noncoherent decision feedback multiuser detection: Optimality, performance bounds, and rules for ordering users," in Proc. 1998 Int. Symp. Inform. Theory, 1998, p. 35.

[11] H. V. Poor and S. Verdu, "Probability of error in MMSE multiuser detection," IEEE Trans. Inform. Theory, vol. 43, pp. 858-871, Mar. 1998

[12] L. L. Scharf and B. Friedlander, "Matched subspace detectors," IEEE Trans. Signal Processing, vol. 42, pp. 2146-2157, Aug. 1994.

[13] M. Schwartz, W. Bennet, and S. Stein, Communication Systems and Techniques. New York, NY: IEEE Press, 1996.

[14] J. G. Proakis, Digital Communications. New York: McGraw-Hill, 1995. 